\title{
Muscle Contractility and Cell Motility
}

\author{
J.-P. Jin, ${ }^{1}$ Robert J. Bloch, ${ }^{2}$ Xupei Huang, ${ }^{3}$ and Lars Larsson ${ }^{4}$ \\ ${ }^{1}$ Department of Physiology, Wayne State University School of Medicine, Detroit, MI 48201, USA \\ ${ }^{2}$ Department of Physiology, University of Maryland School of Medicine, Baltimore, MD 21201, USA \\ ${ }^{3}$ Department of Biomedical Sciences, Florida Atlantic University Charles E. Schmidt College of Medicine, \\ Boca Raton, FL 33431, USA \\ ${ }^{4}$ Department of Neuroscience, Clinical Neurophysiology, Uppsala University, 75185 Uppsala, Sweden
}

Correspondence should be addressed to J.-P. Jin, jjin@med.wayne.edu

Received 30 November 2011; Accepted 30 November 2011

Copyright ( 2012 J.-P. Jin et al. This is an open access article distributed under the Creative Commons Attribution License, which permits unrestricted use, distribution, and reproduction in any medium, provided the original work is properly cited.

Muscle contractility and cell motility play a vital role in various physiological and pathological processes, ranging from animal locomotion to cardiovascular function to myopathies and heart failure. Actin-activated myosin II motors power muscle contraction and non-muscle-cell motility. Actinmyosin-derived contractility has been most extensively studied in striated muscle (skeletal and cardiac muscles), but it is also the foundation for smooth muscle contraction and non-muscle-cell motility. Recent advances in biology and medicine have significantly enriched our understanding of the molecular mechanisms underlying muscle contractility and cell motility.

The generation and regulation of contractility present a natural topic for a special issue of this Journal. Three centuries of scientific investigation since Leeuwenhoek and Croone has led to a detailed understanding of muscle function and the broader field of cell motility, which allows us to understand biological movements, their biochemical and mechanical underpinnings, and their effects on the integrity of muscle tissue in health and disease. Therefore, a challenge of the seemingly straightforward task of editing this special issue is the coverage of the vast areas of research on biological movement. With the enthusiasm of our outstanding contributing authors, this special issue comes with a nice collection of six review articles, 13 original research papers, and one methodology report, covering a broad range of topics in the current research of contractility and muscle function.

Among the six review articles, C.-Y. Tang and T.-Y. Chen discussed the CLC-1 chloride channel, a member of the CLC channel/transporter family, for its roles in the physiological function and diseases of skeletal muscle. The observation that mutations scattered throughout the entire CLC-1 protein result in myotonia congenita, with no clear relationship to the inheritance pattern or channel function, suggests that the pathophysiology of myotonia mutations may involve multiple molecular and cellular mechanisms including channel function or protein synthesis and membrane trafficking mechanisms.

$\mathrm{G}$. Wu and colleagues presented a summary of the planar cell polarity signaling pathway in congenital heart diseases. This pathway, responsible for tissue polarity in Drosophila and gastrulation movements and cardiogenesis in vertebrates, plays multiple roles during cardiac differentiation. Knowledge gained in studies of planar cell polarity signaling has helped investigators understand the pathogenesis of congenital heart diseases.

The review by I. dela Pena and J. H. Cheong, on benzofuroindole analogues as smooth muscle relaxants, focused on the potential therapeutic implications of benzofuroindole compounds in disease states linked to smooth muscle hyperactivity caused by the potentiation of large conductance $\mathrm{Ca}^{2+}$-activated $\mathrm{K}^{+}$channels. The authors also discussed the value of benzofuroindole compounds and the challenges that need to be overcome if they are to be considered as smooth muscle relaxant drugs.

J. Gumerson and D. Michele reviewed the role of the dystrophin-glycoprotein complex in the prevention of muscle damage. Although normally benign in healthy muscle, contractions in dystrophic muscle may contribute to a higher degree of muscle damage, which eventually overwhelms 
the capacity of muscle to regenerate. The dystrophin-glycoprotein complex plays an important function in preventing muscle damage and, when impaired, may contribute to the pathogenesis of muscular dystrophy. It also plays potentially significant roles in signaling pathways that are essential to muscle health.

The review by M. Ackermann and A. KontrogianniKonstantopoulos on myosin-binding protein-C focuses on its role as a regulator of actomyosin interaction in striated muscle. The cardiac, slow skeletal, and fast skeletal muscle isoforms of myosin-binding protein-C contribute to the assembly and stabilization of thick filaments and regulate the formation of actomyosin cross-bridges via direct interactions with both thick myosin and thin actin filaments. Studies on the mutations in cardiac myosin-binding protein- $\mathrm{C}$ associated with familial hypertrophic cardiomyopathy are summarized.

E. Kachaeva and B. S. Shenkman present a review on the functions of proteolytic enzymes in skeletal muscle during unloading. A shift of protein synthesis/proteolysis balance towards increased protein degradation is associated with disuse muscle atrophy. Disuse atrophy of postural muscles caused by unloading differs from that induced by denervation or other conditions. Maintaining of the balance, in which approaches lead to muscle adaptation to disuse rather than muscle degeneration, is discussed with recent data on the activity of related signaling systems.

The thirteen research papers collected in this special issue also covered a broad range of topics. For example, A. McMillan and colleagues studied diffusion tensor magnetic resonance imaging to assess damage in healthy and dystrophic skeletal muscle after injuries caused by lengthening contractions. The aim was to determine if diffusion tensor imaging would serve as a reliable marker of damage after a muscle strain injury in dystrophic and control mice. The study found greater increases in apparent diffusion coefficient and axial and radial diffusivity in injured $m d x$ muscles versus controls. These changes were paralleled by decreases in fractional anisotropy. The data suggest that diffusion tensor magnetic resonance imaging is an accurate and early indicator of muscle injury.

The paper by P. Robison et al. presents a study of adherent primary cultures of mouse intercostal muscle fibers for physiological studies. They described a system that allows for monitoring functional properties of living muscle fibers in culture, with or without electrical-field-stimulated fiber contraction, at physiological or pathological respiratory firing patterns. They also report initial characterization of these fibers, demonstrating several common techniques in this new model system in the context of the established flexor digitorum brevis muscle primary culture model.

I. Ogneva present a study on the transversal stiffness and beta-actin and alpha-actinin- 4 content of rat soleus muscle fibers in 3-day reloading after 14-day gravitational unloading by hindlimb suspension. Analyzing the structural changes in different parts of the sarcolemma and contractile apparatus of muscle fibers by measuring their transversal stiffness by atomic force microscopy, the author showed that 14 days of disuse reduced the transverse stiffness of all points of the sarcolemma and contractile apparatus. Readaptation for 3 days led to complete or partial recovery of the values of the transverse stiffness of the sarcolemma and contractile apparatus, respectively.

P. Zhao and colleagues studied the equal force recovery in dysferlin-deficient and wild-type mouse muscles following saponin exposure. Their study found that dysferlin-deficient muscles recovered tetanic force production to the same extent as wild-type controls following a 5-min saponin exposure. The slow soleus muscle recovered significantly better than the fast extensor digitorum longus muscle. The results suggest that it is unlikely that dysferlin is involved in repairing saponin-induced membrane damage and that slow muscle is more efficient than fast muscle in repairing such damage.

A. L. Vega et al. report the dynamic changes in sarcoplasmic reticulum structure in ventricular cardiomyocytes. They tested the hypothesis that the sarcoplasmic reticulum is a structurally inert organelle in ventricular myocytes. The results suggest that, rather than being static, the sarcoplasmic reticulum undergoes frequent dynamic structural changes, which occurred in the absence of changes in $\left[\mathrm{Ca}^{2+}\right]_{i}$. Microtubules and the molecular motors, dynein and kinesin 1 (Kif5b), were important regulators of the sarcoplasmic reticulum motility.

E. Spangenburg and colleagues studied the use of BODIPY (493/503) dye to visualize intramuscular lipid droplets in skeletal muscle. They found that this dye was a convenient and simple reagent to visualize lipid droplets in both sectioned skeletal muscle and cultured adult single fibers. The dye was effective in both fixed and unfixed cells and was unaffected by permeabilization and thus provides a simple method for visualizing lipid droplets in skeletal muscle.

M. Fiege et al. investigated the in vivo effects of theophylline on anesthetized malignant hyperthermia-susceptible pigs. The results showed that theophylline induced comparable clinical alterations in the anesthetized malignant hyperthermia-susceptible and control swine. No pig developed hypermetabolism and/or malignant hyperthermia. Even at cumulative doses much higher than the doses used therapeutically in humans, theophylline was thus not a trigger of malignant hyperthermia in genetically determined swine.

C. Paolini and coworkers studied the differential effect of calsequestrin ablation on the structure and function of fast and slow skeletal muscle fibers. They found that, in extensor digitorum longus and soleus muscles of adult mice lacking calsequestrin, the main sarcoplasmic reticulum $\mathrm{Ca}^{2+}$ binding proteins induced ultrastructural alterations in $\sim 30 \%$ of soleus fibers but not in extensor digitorum longus fibers. Twitch time parameters were prolonged in both muscles, although tension was not reduced. Calsequestrin is essential in extensor digitorum longus muscle to provide large amount of $\mathrm{Ca}^{2+}$ released from the sarcoplasmic reticulum during tetanic stimulation, whereas soleus muscle tolerated better in the absence of calsequestrin.

L. Zhang and colleagues present a study of the synergistic effects between phosphorylation of phospholamban and cardiac troponin I on promoting cardiac muscle relaxation. 
In isolated working rat hearts, less increases in cardiac output and relaxation rate were found in rapid pacing than that in isoproterenol treatment at the same increments of heart rates. Rapid pacing induced phosphorylation of phospholamban at $\mathrm{Thr}_{17}$, whereas isoproterenol induced phosphorylation of cardiac troponin I and phospholamban at $\operatorname{Ser}_{16}$ and $\mathrm{Thr}_{17}$. The results suggest synergistic effects of phospholamban and cardiac troponin I on diastolic cardiac function to facilitate filling at high heart rate.

The study presented by R. A. Zuellig et al. investigated the tissue expression and actin binding of a novel N-terminal utrophin isoform. Utrophin and dystrophin are two large proteins that link the intracellular actin cytoskeleton to the extracellular matrix via the C-terminal-associated protein complex. The authors report a novel short N-terminal isoform of utrophin and its expression in various rat tissues. The short N-terminal utrophin bound actin with pronounced negative cooperativity and was $\mathrm{Ca}^{2+}$ insensitive. Recombinant short N-terminal utrophin associated to cell membranes, suggesting that it may be responsible for the anchoring of the cortical actin cytoskeleton to the membranes in muscle and other tissues.

S. B. Shah and colleagues present a paper on the influences of desmin and keratin 19 on passive biomechanical properties of mouse skeletal muscle. The authors examined the passive load-bearing response of single fibers from the extensor digitorum longus muscle of 3- and 10-month wildtype, desmin-null, K19-null, and desmin/K19 double-null mice. Though fibers are more compliant in all mutant genotypes compared to wildtype, the structural response of each genotype is distinct, suggesting multiple mechanisms by which desmin and keratin influence the biomechanical properties of myofibers and providing insights into the influences of intermediate filaments on the structure and function of skeletal muscle.

M. Raeker and M. Russell report that obscurin depletion impairs organization of skeletal muscle in developing zebrafish embryos. Using the developing zebrafish embryo as a model, they examined the relationship between new myofibril assembly and the organization of the membrane domains involved in cell-extracellular matrix interactions. The results showed that depletion of obscurin, a giant muscle protein, resulted in irregular cell morphology and disturbed extracellular matrix organization during skeletal muscle development. The impairment suggests that obscurin participates in organizing the internal structure of the myocyte and in regulating the ability of muscle cells to organize the surrounding matrix.

N. Fujita et al. studied the combined effect of electrical stimulation and high-load isometric contraction on protein degradation pathways in muscle atrophy induced by hindlimb unloading. Electrical stimulation alone resulted in slight attenuation of muscle atrophy. Combining electrical stimulation with high-load isometric contraction enhanced this effect. Electrical stimulation alone inhibited the overexpression of calpain 1, calpain 2, and MuRF-1 mRNA. Electrical stimulation with high-load isometric contraction further inhibited the overexpression of cathepsin L and atrogin-1. These findings suggest that the combination of electrical stimulation and high-load isometric contraction may be effective as a countermeasure against muscle atrophy.

To conclude this special issue, the methodology report by B. Biesiadecki and J.-P. Jin describes a high-throughput solidphase microplate protein-binding assay with examples investigating interactions between myofilament proteins. This novel method assesses protein-protein interactions at physiological conditions, utilizes relatively small amounts of protein, is free of protein modification, and does not require specialized instrumentation. Detailed methodology is presented for quantitative studies. Optimization of the assay conditions and its broader application in studies of other protein-protein interactions are also presented.

\section{Acknowledgments}

On behalf of all contributing authors, we would like to thank the JBB Editorial Office for the opportunity of producing this special issue. Our sincerest thanks also go to the outstanding external reviewers for their expert assistance.

J.-P. Jin

Robert J. Bloch Xupei Huang

Lars Larsson 

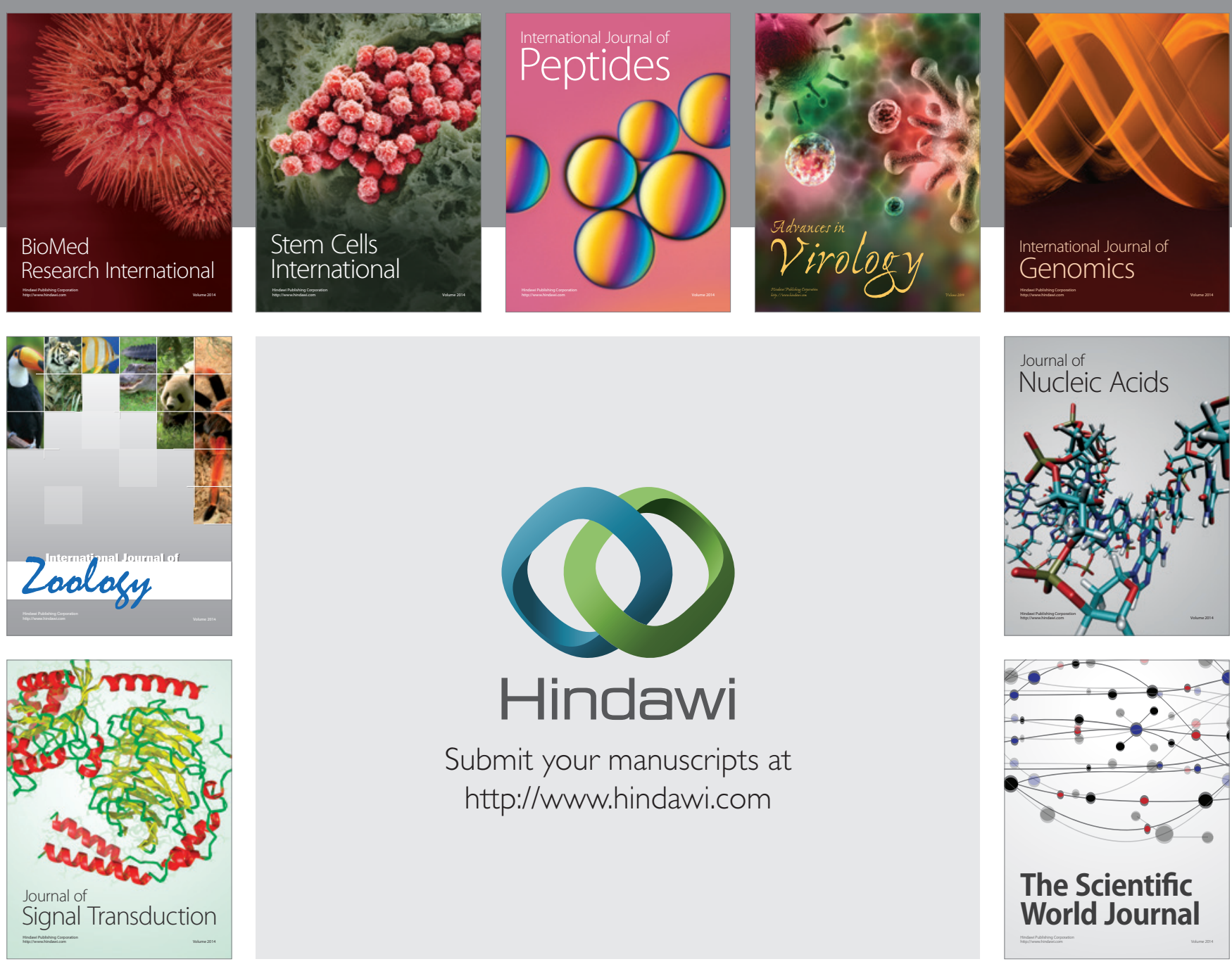

Submit your manuscripts at

http://www.hindawi.com
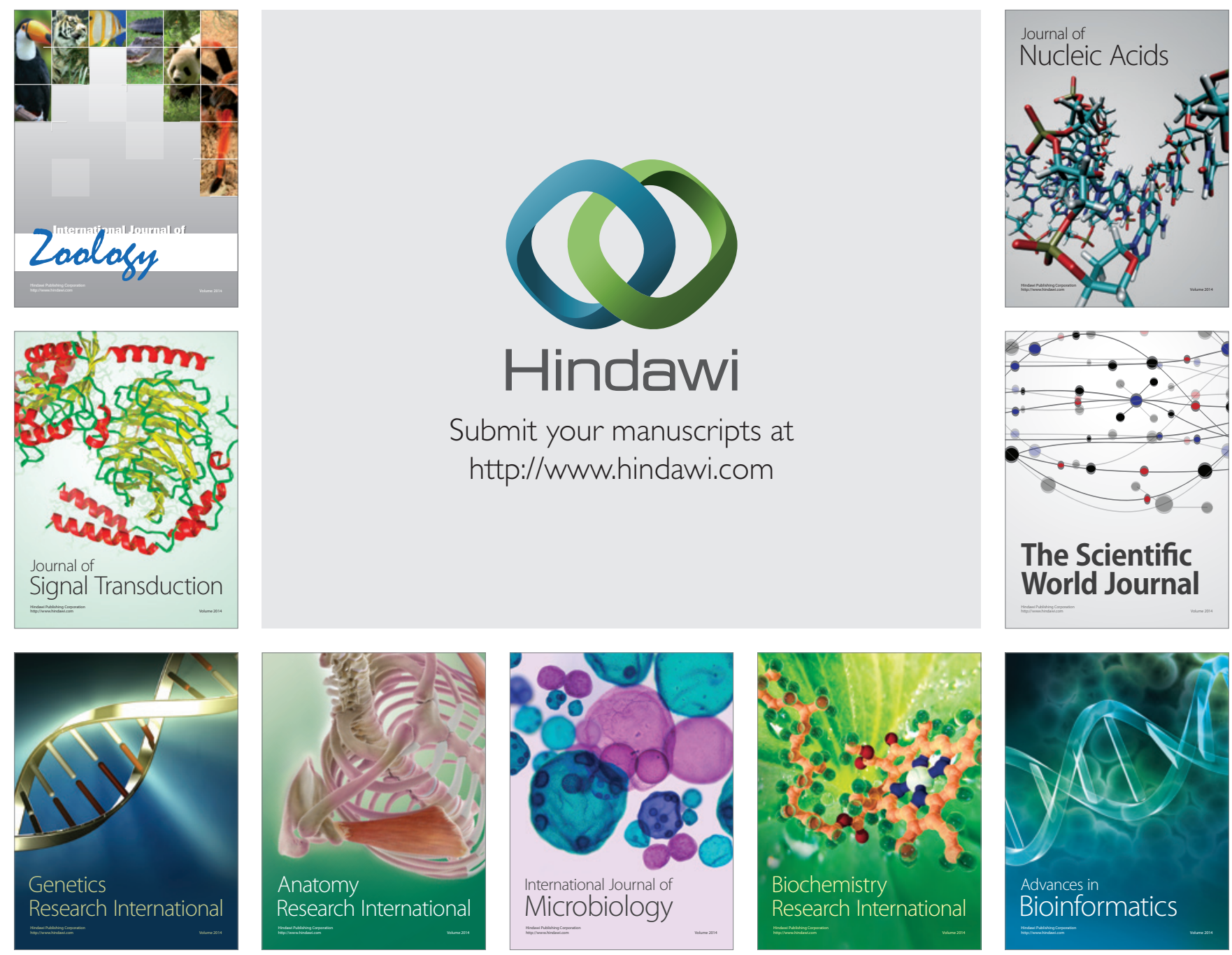

The Scientific World Journal
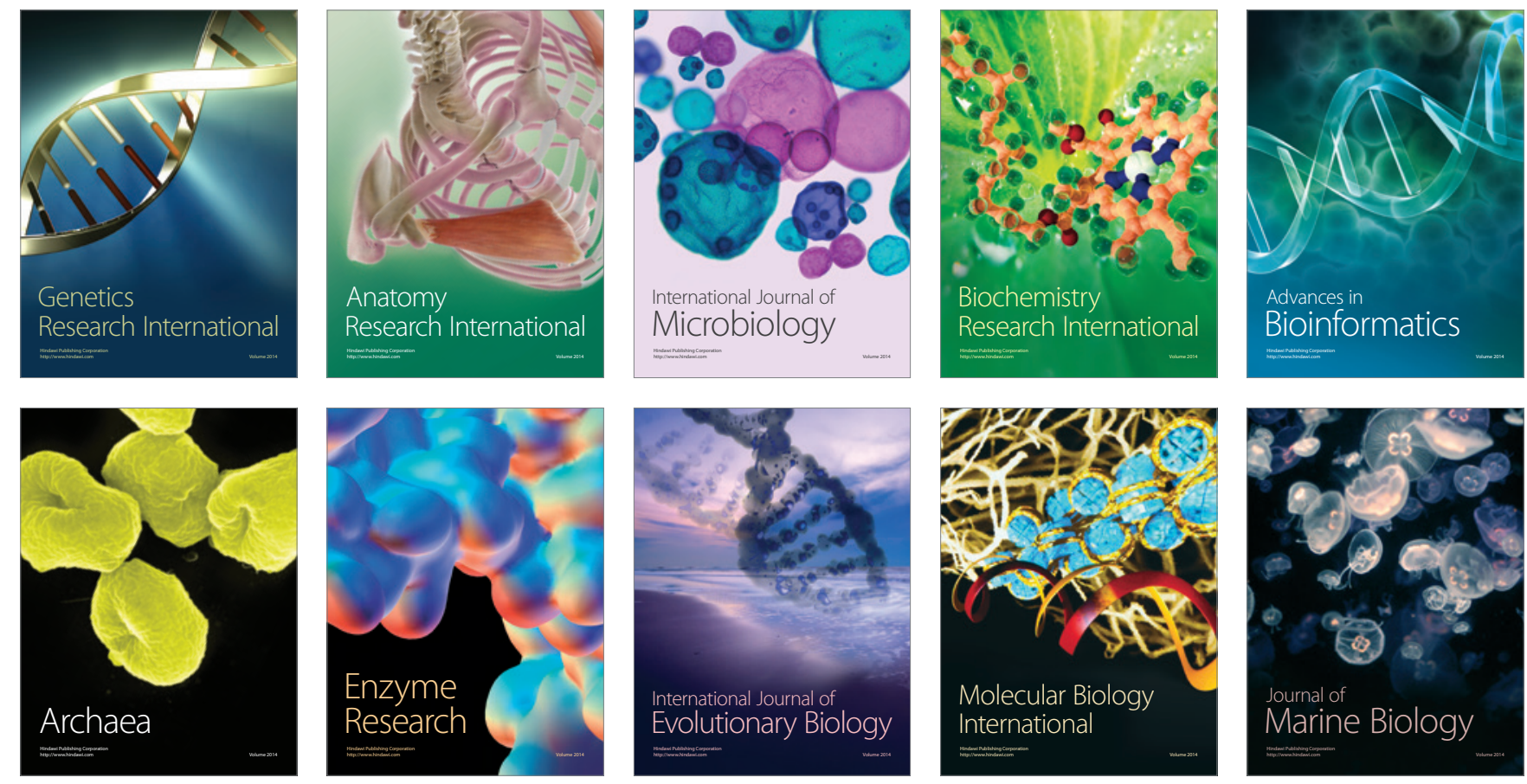